\title{
Optimal manoeuvre for two cooperative military elements under uncertain enemy threat
}

\author{
Dušan Hrabec* \\ Faculty of Applied Informatics, \\ Tomas Bata University in Zlín, \\ Nad Stráněmi 4511, \\ 76005 Zlín, Czech Republic \\ E-mail: hrabec@fai.utb.cz \\ ${ }^{*}$ Corresponding author
}

\section{Jan Mazal and Petr Stodola}

Faculty of Military Leadership, University of Defence,

Kounicova 65, 66210 Brno, Czech Republic

Email: jan.mazal@unob.cz

Email: petr.stodola@unob.cz

\begin{abstract}
Consider an armed military group of two friendly elements that need to move between two given locations - a starting point and an end point - in an environment with the possible presence of armed enemy elements. The potential positions of the enemy may or may not be known in advance. Therefore, we capture the possible threat by identifying the locations that are advantageous from the enemy's perspective. We model the problem using a stochastic programming approach. We then provide a deterministic reformulation of the problem in consideration of this possible threat and, through the use of cooperation, we minimise the total predicted threat as well as the suitability of the terrain for movement (or total length). Finally, we provide concrete experimental calculations and visualisations in our tactical information system, which serves as a tool for commanders to support their decision-making processes, and compare the obtained results to a selection of different approaches.
\end{abstract}

Keywords: Optimal/safe manoeuvre; operations research; mathematical modeling; decision-making; stochastic optimisation; shortest path; military tactics; cooperative military elements.

Biographical notes: Dušan Hrabec received his MSc degree in Mathematical Engineering from Brno University of Technology in 2011. He is a PhD student of Applied Mathematics in the Institute of Mathematics, Faculty of Mechanical Engineering at the Brno University of Technology, Czech Republic. Recently, he works at Department of Mathematics, Faculty of Applied Informatics, Tomas Bata University in Zlín, Czech Republic. His research and developments efforts centre on operational research, stochastic optimization and its applications.

Jan Mazal received his Ph.D. on Military Academy in Brno in the field of the defence management theory and since 2013 he is an associated professor of the 
military management theory. He currently holds the position at the University of Defence in Brno as a chief scientist of the Department of Military Tactics. His research is focused on the issue of military robotics, artificial intelligence, optimal reconnaissance, C4ISR systems and implementation of an advanced modelling and simulation to the commander's decision-making process.

Petr Stodola received his Ph.D. degree in the field of military technology at University of Defence in Brno in 2006. He is currently an associated professor at University of Defence where he works as a senior researcher of Department of Tactics. His research interests include optimization, combat modeling and simulation, command and control decision support systems and C4ISR systems.

\section{Introduction}

With the rise of the future soldier system, an increase in the level of combat decision-making support is needed. Despite the importance of this situation, the literature on optimising a future soldier-commander system for the performance of ground forces is surprisingly sparse (Lee et al., 2014), although support for decision-making in other instances of mission planning is not exceptional; e.g., aircraft (Quttineh and Larsson, 2014) and marine (Bruzzone et al., 2015) systems or military medical evacuation assets (Rettke et al., 2016). Therefore, tactical decision support systems have recently become trends for research in combat management. All modern armies are interested in utilising these new possibilities (Mazal et al., 2012).

Operations research (OR) deals with the application of advanced analytical methods in order to help with better decision-making. The term OR was not used until 1940, when the first results involved the planning of British retaliatory air strikes in Germany, the planning of anti-aircraft protection in England, and, typically, optimising the size as well as the allocation of army units, their supplies, and their transportation (Bastian et al., 2016).

Mathematical programming (MP), and chiefly linear programming (LP), is one of the best developed and most used branches of OR. LP deals with the problem of minimising or maximising linear functions in the presence of linear inequalities. The popularity of LP can be attributed to many factors including its ability to model large and complex problems. A common method is to formulate real-world problems in detailed mathematical models, and subsequently to use techniques for solving the models (algorithms) and engines for executing these algorithms (computers and software) (Dantzig and Thapa, 1997).

In this paper, the methods of MP are applied to solve a problem belonging to the military domain. The paper focuses on the mathematical model dealing with the optimal movement of a two-element military group through an environment in which an armed enemy may be present. The objective is to minimise the threat in tandem with the path length, considering the suitability of the terrain while moving through such an environment. The positions of the enemy may or may not be known in advance. We utilise the cooperation of the friendly elements, i.e., the opportunity for a lurking element to cover the second element during his movements and vice versa until both of these are no longer in the target location. Since, to our best knowledge, there is no existing literature dealing with such a problem, we consider the two-element group as a first step in the research, which leads to a suitable illustrative model. 
Recently, mathematical models underlie computer programs supporting decisionmaking, while bringing order and understanding to the huge flow of data computers produce (Mazal et al., 2015). Therefore, the proposed model has been implemented into our tactical information system, which serves as a tool for commanders to support their decision-making processes. This system includes other models for both simple and advanced military tactics such as strike, fire, ambush, attack, search for an optimal observation post, the optimal supply of units on the battlefield, the optimal reconnaissance of the area of interest via UAVs, as well as other elements.

\subsection{Remarks on the literature}

The model developed in this paper spans several streams of the literature. One of the main streams involves the literature on MP. We further subdivide the area of MP into multiple major fields. The first field consists of the literature on LP (Vanderbei, 2001). Closely linked is the literature on LP and network flows (Bazaraa et al., 2006). A useful topic stemming from this branch includes logistics systems planning and control (Ghiani et al., 2004). The second field of MP presented in this paper consists of problems with a random factor (Birge and Louveaux, 1997) that may lead to stochastic programming (SP) problems. Finally, integer programming (IP) deals with models where some of the variables are discrete (Wolsey, 1998). See also Rahmani et al. (2014) for a combination of the SP approach with the so-called mixed-integer linear programming problem.

A second stream related to this paper is the literature on shortest path-finding approaches; see, e.g., Olya (2014). Since, in the formulation of our problem, the decision for the optimal path (or destination node, alternatively) is under uncertainty (Naini et al., 2013; Chen et al., 2013), we also refer to Lücking and Stadje (2013) for the stochastic shortest-path problem. Beyond these sources, we also refer to the following references: the military unit path-finding problem (Leenen et al., 2012; Rybansky, 2014; Zhanga and Duana, 2015), the problem of finding paths for small groups of characters in an urban environment (Kamphuis et al., 2005), and motion planning techniques (Geraerts and Overmars, 2004). Recently, there is increasing research and development on extensions into intelligent and dynamic systems (Dalfard, 2014; Jose and Zhuang, 2013; Mazal et al., 2015).

There are not many available publications that deal with the concept of the computational support of commanders to help them with their decision-making. Although it is very probable that modern armies are developing such systems and tools at the moment, there is nonetheless a lack of information available on these systems. One exception is the Deep Green project being undertaken by the US Army (Defense Industry Daily, 2008), which is a project financed by the DARPA agency. Deep Green is a system for U.S. Army commanders to help them rapidly generate courses of action (options) through multimodal sketch and speech recognition technologies (Surdu and Kittka, 2008).

\subsection{Our contribution}

We propose a novel approach to the problem of optimal manoeuvre. We model the situation as an SP problem, in which the enemy threat is somehow uncertain. We believe that by combining the MP/SP approach with military tactics and optimal path planning approaches, we can achieve a sensible and high-quality model that can have a significant contribution on the common approaches to the problem. See, e.g., paper by Mazal et al. (2012) for ideas on optimal path planning. Moreover, the formulated model/strategy could by further used 
in various problems (e.g., for a group of cooperative robots or other elements as agents in a computer animation).

In the paper, we will further consider a two-element group (as the first step of the research). Potentially higher groups may then be considered as a logical extension of our model (Kamphuis et al., 2005).

\section{MP problem formulation}

We consider a two-element group that needs to move from a given start point to a given end point. We suppose that we have some environmental knowledge (see section 3), excluding knowledge of enemy positions. Using facts that arise during the manoeuvre, we try to minimise the total risk presented by the enemy threats together with path length as well as path length suitability. Additionally, to protect one element during his movements, coverage is provided by the second element in advance and vice versa until both elements are out of the target location. Therefore, we seek to find the optimal two-element manoeuvre for such a recursive problem.

\subsection{Formulation of problem with a random factor}

From our perspective, the main challenge of such a problem lies in the uncertainty related to the possible positions of the enemy. First of all, we must define the optimisation problem, including uncertainty.

In general, different approaches to modelling problems with uncertainty may be used. The most frequent way to introduce such a problem is to present a deterministic problem and then to claim that some of its data are stochastic (Kall and Wallace, 1994).

Let the triplet $(\Omega, \mathscr{F}, P)$ be a probability space. From there, we can formulate the general problem with a random factor as

$$
\min _{\boldsymbol{x}}\{f(\boldsymbol{x}, \boldsymbol{\xi}) \mid \boldsymbol{g}(\boldsymbol{x}, \boldsymbol{\xi}) \circ \mathbf{0}, \boldsymbol{x} \in C(\boldsymbol{\xi})\},
$$

where $C(\boldsymbol{\xi})$ is a set of feasible solutions defined as

$$
C(\boldsymbol{\xi})=\bigcap_{k=1}^{l} C_{k}(\boldsymbol{\xi})=\bigcap_{k=1}^{l}\left\{\boldsymbol{x} \in X \mid g_{k}(\boldsymbol{x}, \boldsymbol{\xi}) \circ 0 \text { for } 1 \leq k \leq l\right\} .
$$

$C(\boldsymbol{\xi}) \subset \mathbb{R}^{n}$ and o represents a column vector of symbols $\leq, \geq,=($ Popela, 1998, 2010). Then, $f: \mathbb{R}^{n} \times \Omega \rightarrow \mathbb{R}$ is the objective function, a decision $\boldsymbol{x} \in X \subset \mathbb{R}^{n}$ is the variable satisfying the condition of feasibility $\boldsymbol{x} \in C(\boldsymbol{\xi})$ and criterion $f$ and $\boldsymbol{\xi}=\left(\xi_{1}, \ldots, \xi_{m}\right)^{T}$ is a $\mathscr{F}$-measurable mapping $\boldsymbol{\xi}: \Omega \rightarrow \mathbb{R}^{m}$ (it induces a probability distribution on $\mathbb{R}^{m}$ ), and we call it the random vector (random variable) varying over a set $\Xi \subset \mathbb{R}^{m}$. More precisely, we assume all over that a family $\mathscr{F}$ of "events", i.e. subsets of $\Xi$, and the probability distribution $P$ on $\mathscr{F}$ are given. Hence for every subset $A \subset \Xi$ that is an event, i.e. $A \in \mathscr{F}$, the probability $P(A)$ is known. We assume that the functions $g_{k}(\boldsymbol{x}, \cdot): \Xi \rightarrow \mathbb{R}, \forall \boldsymbol{x}, k$, are random variables themselves, and that the probability distribution $P$ is independent of $\boldsymbol{x}$ (Kall and Wallace, 1994).

In our case, $f(\boldsymbol{x}, \boldsymbol{\xi})$ presents a total objective function, with the random variable $\boldsymbol{\xi}$ somehow capturing the threat, e.g. a set of all possible events with a probability that is often given by probability distribution, $\boldsymbol{x} \in C(\boldsymbol{\xi})$ is the decision variable presenting a 
feasible manoeuvre (path), and $\boldsymbol{g}(\boldsymbol{x}, \boldsymbol{\xi}) \circ \mathbf{0}$ presents the system of constraints for a particular problem.

However, the decision on $\boldsymbol{x}$ is undertaken before the realisation of $\boldsymbol{\xi}$ is known; see section 4 for the differences on when the decision is made, i.e., the wait-and-see and hereand-now SP approaches. Therefore, a revision of the modelling process is necessary, leading to deterministic equivalents (Popela, 1998).

Note, that some of the variables could be discrete (e.g., binary or integer). In that case, we prefer to use some of the IP techniques (Wolsey, 1998).

\subsection{Deterministic reformulation}

The main goal of SP problems is to deal with the uncertainty of the possible approach taken (SP theory), which in turns leads to a deterministic equivalent/reformulation that still takes the uncertainty into account (Ruszczynski and Shapiro, 2003; Popela, 1998). However, in our case, the probability distribution of the random variable $\boldsymbol{\xi}$ is not fully known and not enough observations exist; therefore, we utilise some assumptions on the physical environment. We eliminate the uncertainty so that we may establish a suitable placement of enemy locations (from the enemy's perspective) in our environment, i.e., locations

- with good visibility of the nearby environment;

- that are sufficiently hidden.

Note that this approach can remind us of a game in which we try to predict the behaviour of the enemy in order to elucidate the best possible decision-making from our perspective (see, e.g., Von Neumann, 1947).

Moreover, based on the physical attributes (or another available attributes) of these locations, which will be further represented by a vector $\boldsymbol{\xi}$, we determine the suitability for using these placement from the enemy's perspective. We capture this suitability by means of the probability function (see section 2.1). Using a probabilistic approach, we will be able to build the deterministic model (which is given by (2) - (10) in section 3).

\section{Optimal two-element manoeuvre in an environment under enemy threat}

This section provides the final model of the manoeuvre. Before we present the model (subsection 3.6), we determine some useful functions as well as the necessary input data, parameters, indices, and variables (subsections 3.1-3.5).

\subsection{Virtual environment, initial data, and parameters}

For our approach to the problem, we need to determine the virtual representation of the physical environment (or configuration space). We will further work with 2D virtual representation; therefore, we use the set $Y \subset \mathbb{R}^{2}$, where for each point $\left(y_{i} \in Y\right)$ contains the so-called terrain weight $w: Y \rightarrow \mathbb{Z}$. The environment may be also described as the graph $G=(Y, E)$, where $Y$ represents the set of nodes $\left(y_{i} \in Y\right)$ and $E$ is the set of edges, e.g. $\left(y_{1}, y_{2}\right) \in E, y_{1}, y_{2} \in Y$ (Bondy and Murty, 2008), where edge means a connection of nodes. In our setting, every node has 8 edges to the adjacent nodes (Mazal et al., 2012).

To solve this problem, we also need to set an initial (starting) position $y_{s} \in Y$ and a target (end, final) position $y_{f} \in Y$. Then, we define the upper bound of the particular moving 
distance $r$, alternatively a maximal visibility (of the cover element), and two nonnegative increasing functions: a penalty function $p\left(d_{t}\right)$ and a lower-bound-manoeuvre function $l\left(d_{t}\right)$, where both are related to the distance remaining to the target location; see model (2) - (10). We also define the threat weight $v \in \mathbb{Z}$.

\subsection{Distance to the enemy}

We present some suitable functions to capture the suitability of the distance between a shooter and his enemy. The first function is the Gaussian-like function $d_{t}(n)=\alpha e^{-\beta(n-c)^{2}}$, which attains its maximum at $n=c$. The parameters $\alpha$ and $\beta$ represent the scaling and shaping. However, another suitable function derived from the Gumbel probability distribution function (PDF) and which better captures the real situation is:

$$
d_{t}[h(n)]=\frac{1}{\gamma} e^{h+e^{-h}},
$$

where $h(n)=\frac{n-\mu}{\gamma}, \gamma$ is the scale, $\mu$ is the location parameter and $n$ is an Euclidian distance between the friendly and enemy element: $n \geq 0, n=\left\|y_{1}-y_{2}\right\|$. The suitable parameters $\alpha, \beta, \gamma$, and $\mu$ must be used. We use the distance function given by (1) in section 4 . See Figure 1 for more details (Mazal et al., 2011; Mokrá, 2013).

Figure 1: The distance suitability between a shooter and his enemy (Gaussian-like function, $d_{1}$, and Gumbel PDF, $d_{2}$ )
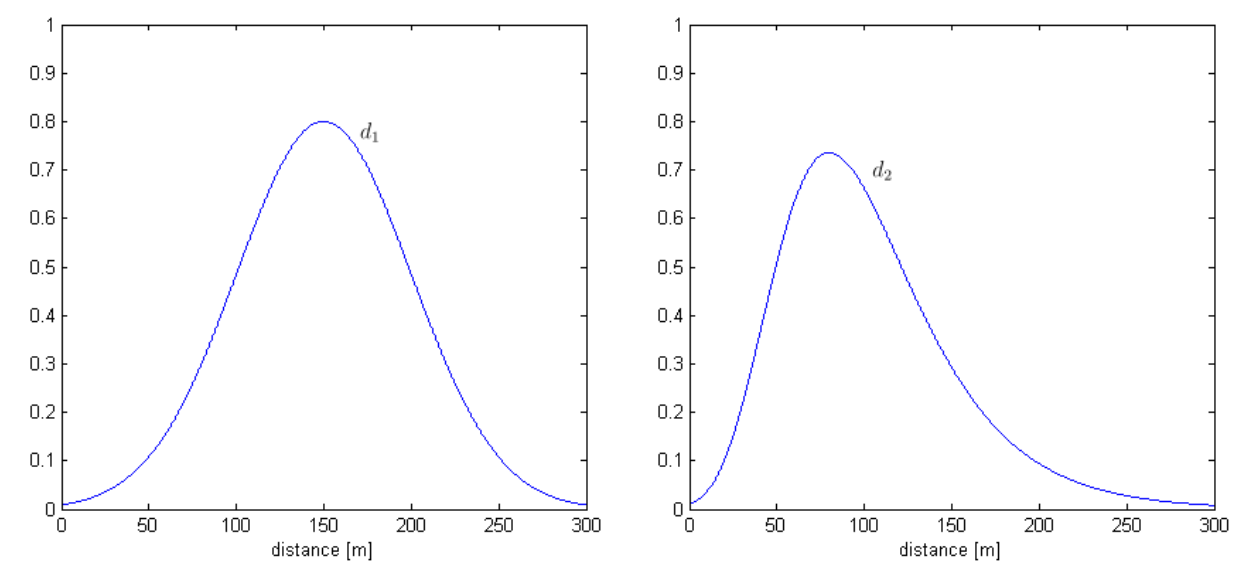

\subsection{Visibility}

We define the visibility function $V\left(y_{i}, y_{j}\right)$, which takes a value from range $[0,1]$ for each pair of points $\left(y_{i}, y_{j}\right), y_{i}, y_{j} \in Y, i \neq j$. For the purpose of this paper, we use only the values 0 and 1 , where $V\left(y_{i}, y_{j}\right)=0$, which means that $y_{j}$ is not visible from $y_{i}$, and where $V\left(y_{i}, y_{j}\right)=1$, which means (full) visibility from $y_{i}$ to $y_{j}$, while the function $V$ is not symmetric. During the manoeuvre, we consider in advance the ability for one element to cover the moving element during his movements, who is not under any threat by an enemy, within the visible area of the covering element. 


\subsection{Indices and uncertain parameters}

The indices are: $t \in T$ representing the set of periods, $t=1,2, \ldots$, then $i \in I$ representing the indices of all points on the optimal path that relate to the period $t$, i.e. $i \equiv i_{t}$ and $i=1,2, \ldots n_{t}$. Moreover, $j \in J$, where $j=1,2, \ldots, m$, where $\xi_{j}$ represents the possible enemy placement/location and $P\left(\xi_{j}\right)$ is its probability.

\subsection{Variables}

The decision variable is $\boldsymbol{x}$, i.e., a sequence of the particular decisions $\boldsymbol{x}_{t}, \boldsymbol{x}=\left\{\boldsymbol{x}_{t}\right\}_{t}=$ $\left(\boldsymbol{x}_{1}, \boldsymbol{x}_{2}, \ldots\right)$, where $\boldsymbol{x}_{t}=\left\{x_{t, i}\right\}_{i}=\left(x_{t, 1}, x_{t, 2}, \ldots, x_{t, n_{t}}\right)$ and $x_{t, i} \in Y \forall t, i$, and so we denote $x_{t, n_{t}}$ as the final position of the moving element in period $t$. Note, that the dimension of the decision variables depends on the decision itself. Moreover, $z_{t}$ denotes the position of the covering element during the period $t, \boldsymbol{z}=\left\{z_{t}\right\}_{t}$. Let $z_{0}=y_{s}$ and $y_{0, n_{0}}=y_{s}$.

\subsection{Model}

As a result, the final deterministic problem to be solved is formulated by:

$$
\begin{array}{rlrl}
\min _{\boldsymbol{x}} \sum_{t \in T}\left[\sum_{i \in I}\left(\sum_{j \in J} P\left(\xi_{j}\right) d\left(n_{i, j}\right) V\left(\xi_{j}, x_{t, i}\right)\left[1-V\left(z_{t}, \xi_{j}\right)\right] v+w_{i}\right)+p\left(d_{t}\right)\right] & & \\
n_{i, j} & =\left\|x_{t, i}-\xi_{j}\right\|, & & \forall i \in I, j \in J, t \in T, \\
z_{t} & =x_{t-1, n_{t-1}}, & & \forall t \in T, \\
x_{t, 0} & =z_{t-1}, & & \forall t \in T, \\
\text { s.t. } & & \forall t \in T, \\
V\left(x_{t, n_{t}}, z_{t}\right) & \geq 1, & \forall t \in T, \\
d_{t} & =\left\|x_{t, n_{t}}-y_{f}\right\|, & & \forall t \in T, \\
\left\|x_{t, n_{t}}-z_{t}\right\| & \leq r, & \forall t \in T, \\
\left\|x_{t, n_{t}}-x_{t, 0}\right\| & \geq l\left(d_{t}\right), & & \forall i \in I, j \in J, t \in T .
\end{array}
$$

The objective function (2) minimises the total threat plus terrain weight (represented by the expression within the round brackets) plus a penalty for the distance remaining to the target position (square brackets); for a more detailed description, see the description of the three various problems in the first part of section 4. Eq. (3) is the Euclidian distance between the point $x_{t, i}$ (i.e., point of the manoeuvre) and possible enemy location $\xi_{j}$. Eq. (4) states the position of the covering element (as the last point of the manoeuvre from the preceding period $t-1$ ), while (5) states the initial point for manoeuvre in period $t$ (as the position of the covering element from the preceding period). (6) is the visibility constraint, i.e., the end point of a particular manoeuvre must be visible to the covering element. (7) states the Euclidian distance between the end point of a particular manoeuvre and the target point $y_{f}$ (of the entire manoeuvre/operation); the distance is used in the penalty function $p\left(d_{t}\right)$ as well as in the lower-bound-manoeuvre function $l\left(d_{t}\right)$. (8) guarantees the maximal distance in each particular manoeuvre. while (9) guarantees that a manoeuvre (with non-zero distance) will be realised. Finally, (10) states the domains of the (decision and random) variables.

We then implement model (2) - (10) into our tactical information system (see Figure $2 \mathrm{~b}$ ). Due to the formulated constraints, especially with suitable $p$ and $l$ functions, we can solve the problem by using a modified Floyd-Warshall algorithm, which has already been presented in the paper by Mazal et al. (2012), see section 4. 


\section{Computational analysis}

In this section, we verify the functionality of model (2) - (10). First of all, we need to determine some necessary inputs for the model: a) the starting point and end location for the manoeuvre, and b) the most probable placement of the enemy elements; see subsection 2.2. See also Figure $2 \mathrm{a}$ for one such enemy placement setting and the related visibilities.

Figure 2: Example of predicted enemy placement, starting point, end location and the solution proposed by the model (2) - (10)

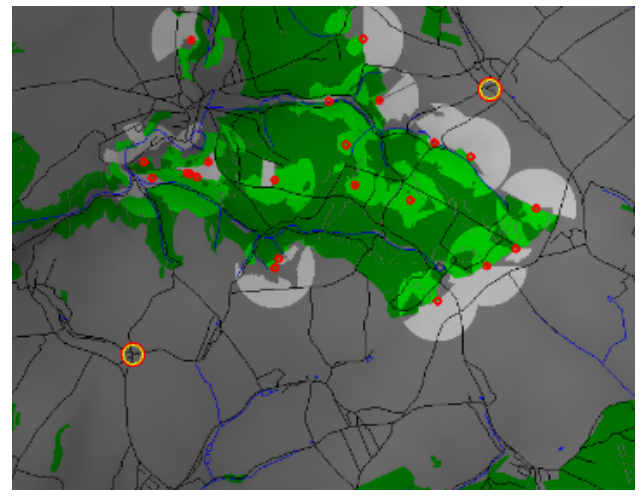

(a) Enemy placement and visibilities

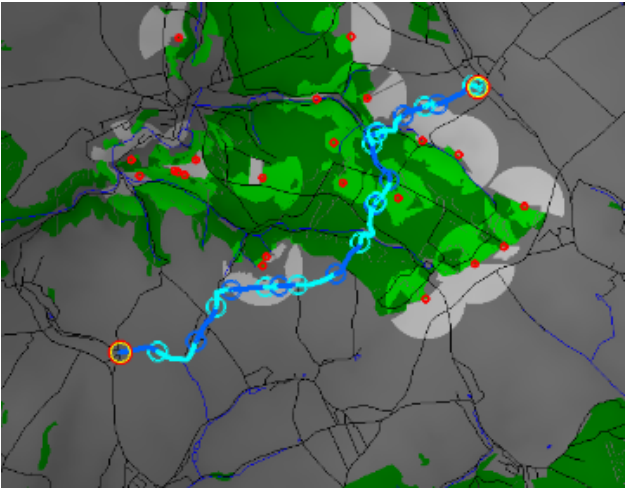

(b) Optimal manoeuvre: model (2) - (10)

\subsection{Various approaches to the manoeuvre}

Further, for the purpose of this section that is to compare the obtained results (or to verify suitability of our approach), we present the following various approaches to the problem:

1. Optimal manoeuvre. In fact, this is model (2) - (10) themselves. Using the formula $\left[1-V\left(z_{t}, \xi_{j}\right)\right]$, we take into account the covering ability of friendly elements. The penalty function, $p\left(d_{t}\right)$, in the model (2) - (10) objective, forces the moving element to move closer to the target location. The manoeuvre is illustrated by the blue piecewise line in Figures $2 b$ and $3 b$, where the paths of the first and the second element as well as their particular covering positions are distinguished in light and dark shades of blue.

2. Stochastic shortest path problem. The first (commonly used) problem to compare is a modification of the so-called stochastic shortest path between two points; see, e.g., Zhan (1997) for an overview of the shortest path algorithms and, e.g., Lücking and Stadje (2013) for the stochastic approach to such algorithms. Stochastic shortest path algorithm, which should be equivalent to our optimal manoeuvre, takes into consideration the sum of the threat (evaluation) multiplied by its probability plus the weight, which is given by:

$$
\sum_{i}\left(\sum_{j} P\left(\xi_{j}\right) d\left(n_{i, j}\right) V\left(\xi_{j}, x_{t, i}\right) v+w_{i}\right), t=1,2, \ldots
$$


The shortest path obtained between the initial and final positions is illustrated by the red piecewise line in Figure 3. This case considers the manoeuvre of only one element through an environment while under the threat. See also Mazal et al. (2012) for the concrete shortest path algorithm that is utilised for the computations here.

3. Empirical manoeuvre. To verify the real suitability of model (2) - (10), we use an empirical solution suggested by experts at the Brno University of Defence. With the knowledge of the predicted placement of enemy locations and their related visibilities, the experts determine concrete, particular covering positions for friendly elements and use the shortest path algorithm between these positions. We present this problem as the empirical manoeuvre and it is illustrated for the first and the second element by the yellow and the orange piecewise lines; see Figure $3 \mathrm{~b}$.

Figure 3: Different approaches to the manoeuvre problem

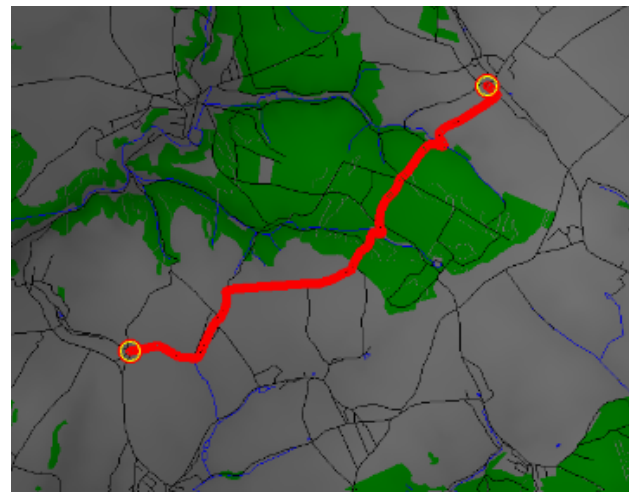

(a) The shortest path without enemy presence

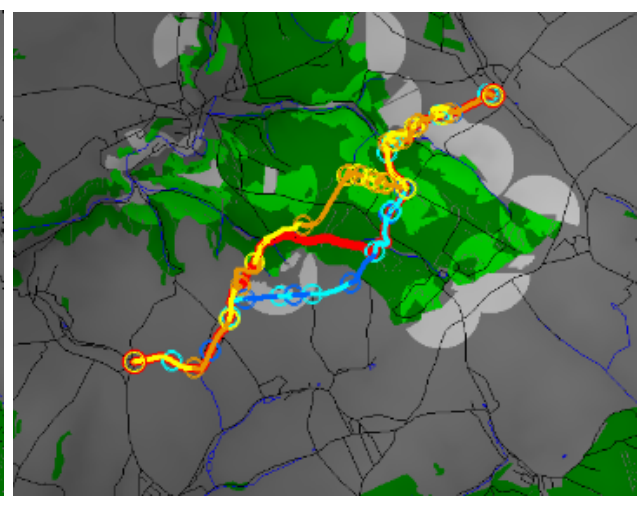

(b) State of Figure 2: three different approaches

\subsection{Computational results and comparison}

We gradually generate 1,3 , and 5 concrete locations from the above-predicted placements of enemy locations (see Figure 4); for each of these cases, we further employ the techniques defined above with two different stochastic approaches:

A. Wait-and-see (WS) approach - 'with knowledge'. With knowledge of the enemy placement (see Figures $4 \mathrm{a}, 4 \mathrm{c}$, and $4 \mathrm{e}$ ), we provide two solutions: the (stochastic) shortest path and the optimal manoeuvre; both are 'reactions' to known enemy positions, i.e., the WS approach to the SP theory (Popela, 1998). In fact, this means that there is no uncertainty in the problem, i.e., $P\left(x_{j}\right)=1$ for all of the considered $j$ in both models used;

B. Here-and-now (HN) approach - 'without knowledge'. Without knowledge of the enemy placement (see Figures $4 \mathrm{~b}, 4 \mathrm{~d}$, and 4f), we firstly determine the path/manoeuvre considering the predicted placement of enemy locations (as in Figure 3b). After that, when the manoeuvre is realised, we compute the 'real' value of the related objective function such that 'there were actually 1,3 , or 5 concrete enemies in the 
environment'. Here, we provide three such solutions: the stochastic shortest path, the optimal manoeuvre, and the empirical manoeuvre. All decisions are made before the final enemy placement is known, i.e., the HN approach to the SP theory (Popela, 1998).

The graphical results obtained are provided in Figure 4. In Table 1, we provide computational results for $0,1,3$, and 5 simultaneous concrete enemy locations. The 'WS' optimal manoeuvre indicates problem 1 with knowledge of enemy placement (case A.), while ' $H N$ ' indicates a lack of such knowledge (case B.). Then, the stochastic shortest path is presented in problem 2 and, finally, the empirical manoeuvre is presented in problem 3 . The values in Table 1 represent the particular values of the related objective functions.

Table 1 Computational results - objective function values

\begin{tabular}{|c|c|c|c|c|c|}
\hline \multirow{2}{*}{$\begin{array}{l}\text { Number } \\
\text { of enemy } \\
\text { elements }\end{array}$} & \multicolumn{2}{|c|}{$\begin{array}{l}\text { 1. Optimal } \\
\text { manoeuvre }\end{array}$} & \multicolumn{2}{|c|}{$\begin{array}{l}\text { 2. Stochastic } \\
\text { shortest path }\end{array}$} & $\begin{array}{l}\text { 3. Empirical } \\
\text { manoeuvre }\end{array}$ \\
\hline & A. WS & B. HN & A. WS & B. HN & B. HN \\
\hline 0 & 14333 & 15447 & 14333 & 15814 & 15860 \\
\hline 1 & 14953 & 15476 & 15766 & 16232 & 15980 \\
\hline 3 & 15605 & 16506 & 17382 & 17718 & 17242 \\
\hline 5 & 16174 & 16539 & 18861 & 19234 & 17368 \\
\hline
\end{tabular}

Note: Since for the stochastic shortest path case, we count the objective function values for only one element, while in the manoeuvre cases we consider two friendly elements; for both the optimal and empirical manoeuvre cases, we provide average values per one element.

We can see from Table 1 that model (2) - (10) as represented by the optimal manoeuvre column, which has been developed throughout this paper, improves the values of the objective function in all particular-comparable cases. Note that we do not compare the WS and $\mathrm{HN}$ approaches themselves. The best value for each row and each stochastic approach is 'framed'. From a practical point of view, the $H N$ case is more realistic ('blue' color in the table), because we do not know for sure what will happen in the future, and so the related values of the objective function are higher (worse) than in all the WS cases.

In real military problems, the shortest path and empirical manoeuvre approaches are typically used. Thus, we may see that the suitable cooperation within a group of friendly elements supported by information from this computational system can significantly improve the objective function value for this type of manoeuvre problem.

\section{Conclusions and further research}

Tactical decision support systems have been increasingly used in contemporary operations on various levels (tactic, operational, strategic). The basic principle consists of the modelling and simulation of military tactics. The goal is to support the decision-making process of commanders on the battlefield via the use of modern technology. The future military command system should conduct intelligence, information, and, especially, security operations These systems are designed for the effective and precise prediction of possible scenarios presented by the situation at hand. Commanders have a better chance of judging the 
Figure 4: WS and HN approaches solved with shortest path (red), optimal (blue), and empirical manoeuvres (yellow) considering 1,3 , and 5 enemy locations

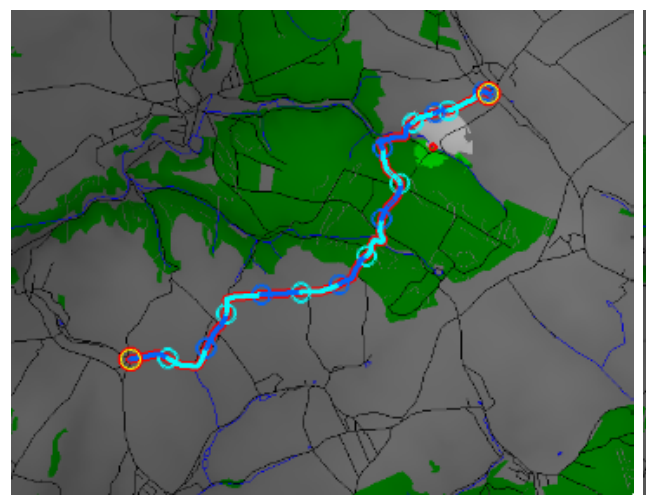

(a) WS approach, 1 enemy location

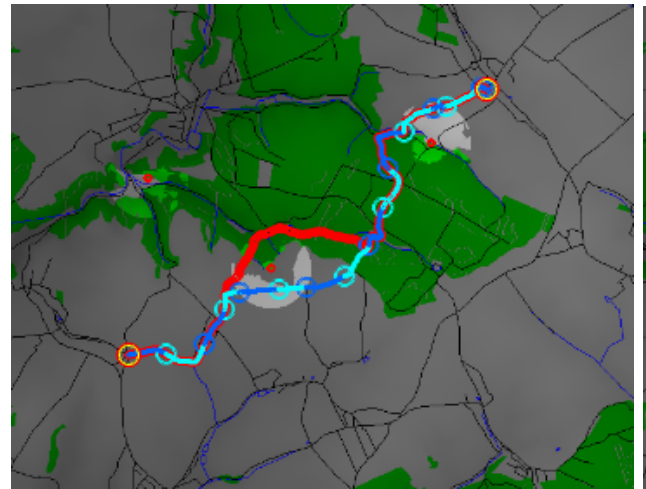

(c) WS approach, 3 enemy locations

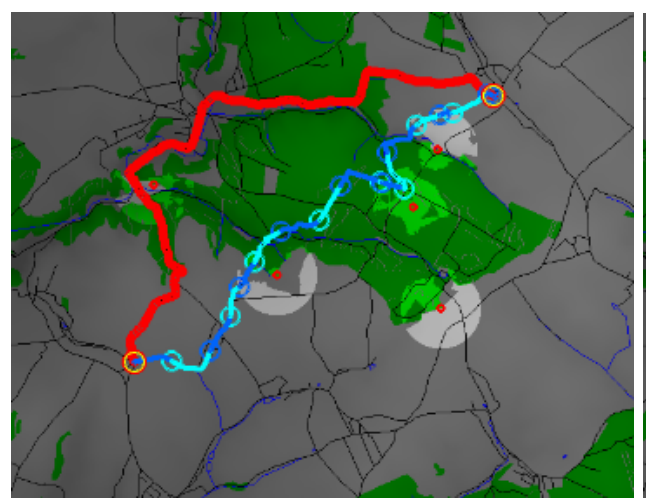

(e) WS approach, 5 enemy locations

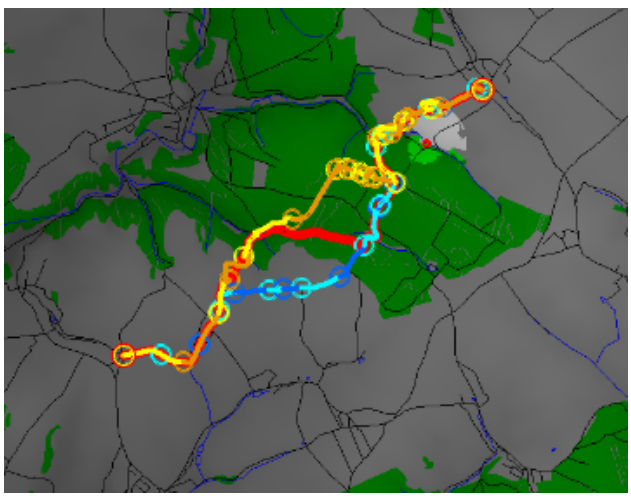

(b) HN approach, 1 enemy location

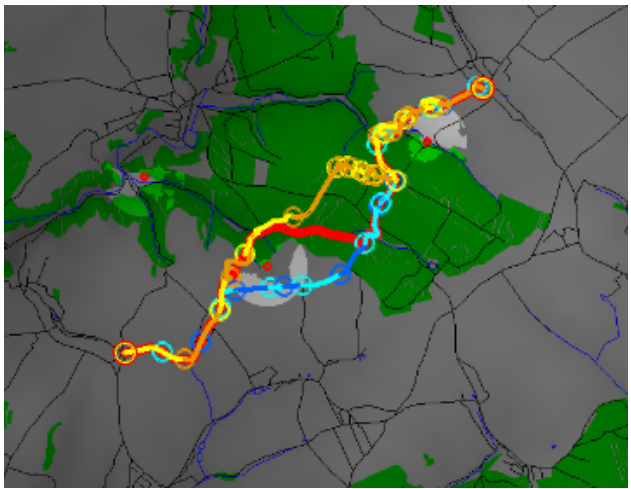

(d) HN approach, 3 enemy locations

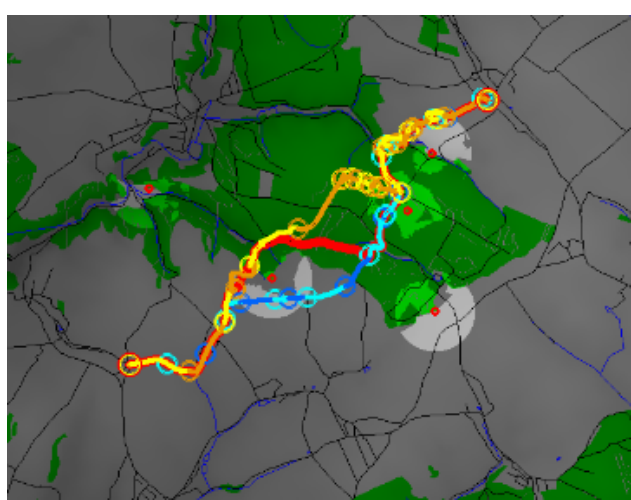

(f) $\mathrm{HN}$ approach, 5 enemy locations

actual situation correctly and evaluating the potential results and impacts of their decisions. 
The advanced prediction ability of these systems is based on the thorough analysis of a current situation with regards to a great number of input parameters.

This article presents a tactical model that may support the decision-making of (military) commanders, with a particular focus on the security and reliability of combat operations. Therefore, the goal of the manoeuvre considered was to minimise the objective function, which is a combination of the enemy threat, manoeuvre path length, and terrain suitability when moving through an environment in which enemy elements may be hidden. The threat is reduced by the cooperation of two friendly elements. The developed model is implemented into the tactical information system and tested by comparing various approaches. We conducted a series of experiments that verified the functionality of the proposed model.

We conclude that using a suitable mathematical model within an information computational system can significantly improve the objective of a given military operation. The objective function value of the manoeuvre considering a group of military elements, even if some of the environmental aspects are somehow uncertain, is not exceptional. We have shown that well-coordinated cooperation (realised via suitable decision-making) within a group can increase the safety of the friendly elements being considered.

On the other hand, further research into the proposed model is called for. There is especially a need to test suitable-effective heuristic solvers on large scale or more complex problem modifications (Zhanga and Duana, 2015). Since similar military methods call for quick decisions, the exact methods are not usually effective (regarding the consumption of time). As previously mentioned, for the next steps in the direction of the research, the problem may (and probably should) be tested for a higher number of cooperative elements (Kamphuis et al., 2005); in this case statistical tests will also be needed for the planned simulations (Michálek et al., 2005). The developed model may also be further used/modified within groups of cooperative elements of robots, agents, or various characters in order to improve their decision-making processes.

\section{Acknowledgements}

This work was supported by the Ministry of Education, Youth and Sports of the Czech Republic within the National Sustainability Programme project No. LO1303 (MSMT7778/2014), and also by the European Regional Development Fund under the project CEBIA-Tech No. CZ.1.05/2.1.00/03.0089.

\section{References}

Bastian, N.D., Griffin, P.M., Spero, E. and Fulton, L.V. (2016) 'Multi-criteria logistics modeling for military humanitarian assistance and disaster relief aerial delivery operations', Optimization Letters, Vol. 10, No. 5, pp.921-953.

Bazaraa, M.S., Sherali, H.D. and Shetty, C.M. (2006) Nonlinear Programming: Theory and Algorithms, $3^{\text {rd }}$ ed., John Wiley and Sons.

Birge, J.R. and Louveaux, F. (1997) Introduction to Stochastic Programming, SpringerVerlag, New York.

Bondy, A. and Murty, U.S.R. (2008) Graph Theory, Graduate Texts in Mathematics, Springer. 
Bruzzone, A.G., Massei, M., Poggi, S., Bartolucci, C. and Ferrando, A. (2015) 'Discrete event simulation for virtual experimentation on marine decision support systems', 17th International Conference on Harbor, Maritime and Multimodal Logistics Modelling and Simulation, HMS 2015, pp.109-116.

Chen, B.Y., Lam, W.H.K., Sumalee, A., Li, Q., Shao, H. and Fang, Z. (2013) 'Finding reliable shortest paths in road networks under uncertainty', Networks and Spatial Economics, Vol. 13, No. 2, pp.123-148.

Dalfard, V.M. (2014) 'A new intelligence algorithm for determination of shortest path for dynamic guidance of vehicles based on service level criterion', International Journal of Operational Research, Vol. 19, No. 4, pp.497-512.

Dantzig, G.B. and Thapa, M.N. (1997) Linear Programming, 1: Introduction, Springer Series in Operations Research.

Defense Industry Daily (2008): DARPA's Commander's Aid: From OODA (Observe, Orient, Decide, Act) to Deep Green, Vermont, Watershed Publishing. Available at: http://www.defenseindustrydaily.com/darpa-from-ooda-to-deep-green-03497/.

Geraerts, R. and Overmars, M.H. (2004) 'A comparative study of probabilistic roadmap planners', Algorithmic Foundations of Robotics V, Vol. 7, pp.43-58.

Ghiani, G., Laporte, G. and Musmanno, R. (2004) Introduction to Logistics Systems Planning and Control, John Wiley and Sons.

Jose, V.R.R. and Zhuang, J. (2013) 'Technology adoption, accumulation, and competition in multiperiod attacker-defender games', Military Operations Research, Vol. 18, No. 2, pp.33-47.

Kall, P. and Wallace, S.W. (1994) Stochastic Programming, John Wiley and Sons.

Kamphuis, A., Rook, M. and Overmars, M.H. (2005) 'Tactical Path Finding in Urban Environments', in First International Workshop on Crowd Simulation (V-CROWDS 05), Lausanne, Switzerland.

Kavraki, L.E., Švestka, P., Latombe, J.-C. and Overmars, M.H. (1996) 'Probabilistic roadmaps for path planning in high-dimensional configuration spaces', IEEE Transactions on Robotics and Automation, Vol. 12, No. 4, pp.566-580.

Latombe J.-C. (1991) Robot Motion Planning, Kluwer Academic Publishers.

Lee, I., Geum, Y., Hong, S.-P., Lee, S. and Choe, E.-J. (2014) 'Optimization of the future soldier system: An integrative approach based on an agent-based simulation and response surface method', Military Operations Research, Vol. 19, No. 4, pp.25-39.

Leenen, L., Vorster, J. and le Roux, W.H. (2012) 'A constraint-based solver for the military unit path finding problem', Mobile Intelligent Autonomous Systems: Recent Advances, pp.225-240.

Lücking, D. and Stadje, W. (2013) 'The stochastic shortest-path problem for Markov chains with infinite state space with applications to nearest-neighbor lattice chains', Mathematical Methods of Operations Research, Vol. 77, No. 2, pp.239-264. 
Mazal, J., Stodola, P. and Podhorec, M. (2012) 'Traffic Management Optimization and its Modeling', Recent Advances in Energy, Environment and Economic Development, pp.274-279

Mazal, J., Stodola, P. Hrabec, D., Kutěj, L., Podhorec, M. and Křištálová, D (2015) 'Mathematical modeling and optimization of the tactical entity defensive engagement', International Journal of Mathematical Models and Methods in Applied Sciences, Vol. 9, pp.600-606

Mazal, J., Stodola, P. and Mokrá, I. (2011) 'Tactical and autonomous UGV navigation', Mechatronics: Recent Technological and Scientific Advances, pp.439-443

Michálek, J, Sedlačík, M. and Doudová, L. (2005) 'A comparison of two parametric ROC curves estimators in binormal model', In Proceedings of the 23rd International Conference Mathematical methods in Economics 2005, pp.256-261

Mokrá, I. (2013) Model Approach to Decision-making Activities of Commanders of Tactical Units in Military Operations, Dissertation (in Czech), University of Defence, Brno

Naini, S.G.J., Paydar, M.M., Jouzdani, J. and Fathian, M. (2013) 'Fuzzy stochastic linear programming-based approach for multiple departures single destination multiple travelling salesman problem', International Journal of Operational Research, Vol. 17, No. 4, pp.417-435.

Olya, M.H. (2014) 'Applying Dijkstra's algorithm for general shortest path problem with normal probability distribution arc length', International Journal of Operational Research, Vol. 21, No. 2, pp.143-154.

Quttineh, N.-H. and Larsson, T. (2014) 'Military aircraft mission planning: Efficient modelbased metaheuristic approaches', Optimization Letters, Vol. 9, No. 8, pp.1625-1639.

Von Neumann, J. and Morgenstern, O. (1947) Theory of Games and Economic Behavior, Princeton University Press.

Popela, P. (1998) An Object-Oriented Approach to Multistage Stochastic Programming: Models and Algorithms, Dissertation, Charles University, Prague.

Popela, P. (2010) 'Stochastic programming models for engineering design problems', Engineering Mechanics, Vol. 17, No. 5-6, pp.351-362.

Rahmani, D., Ramezanian, R. and Saidi-Mehrabad, M. (2014) 'Multi-objective flow shop scheduling problem with stochastic parameters: Fuzzy goal programming approach', International Journal of Operational Research, Vol. 21, No. 3, pp.322-340.

Rettke, A.J., Robbins, M.J. and Lunday, B.J. (2016) 'Approximate dynamic programming for the dispatch of military medical evacuation assets', European Journal of Operational Research, Vol. 254, pp.824-839.

Ruszczynski, A. and Shapiro, A. (2003) Stochastic Programming, Handbooks in Operations Research and Management Science, Vol. 10, Elsevier.

Rybansky, M. (2014) 'Modelling of the optimal vehicle route in terrain in emergency situations using GIS data', IOP Conference Series: Earth and Environmental Science, Vol. 18, pp.1-3. 
Surdu, J. R. and Kittka, K. (2008) The Deep Green Concept, Arlington, DARPA Information Processing Technology Office.

Tarapata, Z. (2003) 'Military route planning in battlefield simulation: effectiveness problems and potential solutions', Journal of Telecommunications and Information Technology, Vol. 2003, No. 4, pp.47-56.

Vanderbei, R.J. (2001) Linear Programming: Foundations and Extensions, Princeton University, New Jersey.

Wolsey, L.A. (1998) Integer Programming, Wiley-interscience Series in Discrete Mathematics and Optimization.

Zhan, F.B. (1997) 'Three fastest shortest path algorithms on real road networks: Data structures and procedures', Journal of Geographic Information and Decision Analysis, Vol. 1, No. 1, pp.70-82.

Zhanga, X. and Duana, H. (2015) 'An improved constrained differential evolution algorithm for unmanned aerial vehicle global route planning', Applied Soft Computing, Vol. 26, pp.270-284. 